\title{
Operationalisierte, kriteriengeleitete Beurteilung der Schuldfähigkeit bei paraphilen Störungen - Stand und Ausblick
}

\author{
S. Dobbrunz' ${ }^{1}$ P. Briken ${ }^{1}$ \\ Eingegangen: 3. Oktober 2020 / Angenommen: 5. Oktober 2020 / Online publiziert: 28. Oktober 2020 \\ ○ Der/die Autor(en) 2020
}

\section{Zusammenfassung}

Die vorliegende Arbeit beschäftigt sich mit der operationalisierten, kriteriengeleiteten Beurteilung für die Begutachtung der Schuldfähigkeit bei paraphilen Störungen. Zunächst werden frühere Überlegungen und Ansätze dargestellt und um neue empirische, vorläufige Ergebnisse, welche erste Annahmen zu Reliabilität und Validität des Vorgehens ermöglichen, erweitert. Anschließend werden kurz die Ergebnisse einer Expertendiskussion zum Thema ergänzt, um vorläufige Schlussfolgerungen und einen Ausblick darzustellen. Insgesamt wird mit der operationalisierten, kriteriengeleiteten Beurteilung für die Begutachtung der Schuldfähigkeit bei paraphilen Störungen ein Verfahren vorgeschlagen, das als vielversprechend angesehen werden kann und zu einer Erweiterung des Vorgehens, den weiteren interdisziplinären Dialog und die weitere empirische Prüfung animiert.

Schlüsselwörter Begutachtung $\cdot$ Sexualstraftäter $\cdot$ Steuerungsfähigkeit $\cdot$ Schuldfähigkeit $\cdot$ Sexuelle Gewalt

\section{Operationalized, criteria-based assessment of culpability in paraphilic disorders-Status and future}

\begin{abstract}
This article describes the operationalized, criteria-based evaluation of culpability in paraphilic disorders. Firstly, previous forms of assessment are presented and extended by new empirical preliminary results, which allow first assumptions about reliability and validity of the proposed procedure. Secondly, the results of an expert discussion on the topic are briefly summarized to present preliminary conclusions and an outlook. The operationalized, criteria-based assessment for the evaluation of culpability in paraphilic disorders proposes a procedure that can be regarded as promising and encourages an expansion of the procedure, further interdisciplinary dialogue and further empirical testing.
\end{abstract}

Keywords Psychiatric expert opinion $\cdot$ Sexual offender $\cdot$ Assessment $\cdot$ Criminal responsibility $\cdot$ Sexual violence

\section{Ausgangslage}

Laut $\S 20$ des Strafgesetzbuches (StGB), ,handelt ohne Schuld, wer bei Begehung der Tat wegen einer krankhaften seelischen Störung, wegen einer tiefgreifenden Bewusstseinsstörung, wegen Schwachsinns oder einer schweren anderen seelischen Abartigkeit unfähig ist, das Unrecht der

Prof. Dr. med. P. Briken

briken@uke.de

1 Institut für Sexualforschung, Sexualmedizin \& Forensische Psychiatrie, Universitätsklinikum Hamburg-Eppendorf, Martinistraße 52, 20246 Hamburg, Deutschland
Tat einzusehen oder nach dieser Einsicht zu handeln“. „Ist die Fähigkeit des Täters, das Unrecht der Tat einzusehen oder nach dieser Einsicht zu handeln, aus einem der in $\$ 20$ bezeichneten Gründe bei Begehung der Tat erheblich vermindert, so kann die Strafe gemildert werden " ( $21 \mathrm{StGB})$. Schuldfähigkeit ist im Gesetzestext nicht positiv definiert, sondern wird ab einem bestimmten Alter als gegeben vorausgesetzt. Der Gesetzgeber gibt vielmehr psychische Zustände oder Situationen vor, in denen die Schuldfähigkeit gemindert oder aufgehoben sein kann. Dazu zählen die Rechtsbegriffe der:

- krankhaften seelischen Störung,

- tiefgreifenden Bewusstseinsstörung, 
- des sog. Schwachsinns ${ }^{1}$ oder

- der schweren anderen seelischen Abartigkeit ${ }^{2}$ (SASA).

Die Einschätzung der Schuldfähigkeit fußt dabei auf der zweistufigen „psychisch-normativen“ Methode (Jescheck und Weigend 1996). Im ersten Schritt erfolgt eine mögliche Zuordnung zu einem der 4 Eingangskriterien des $\S 20 \mathrm{StGB}$, wobei auch ein Zusammenwirken mehrerer psychischer Störungen infrage kommen kann und ein $\mathrm{Zu}$ sammenwirken selbiger somit die Schwelle der ersten Stufe zu nehmen in der Lage ist. Im zweiten Schritt werden Auswirkungen der eben genannten Zustände auf die Einsichtsund Steuerungsfähigkeit untersucht. Die Frage der Schuldfähigkeit bzw. der strafrechtlichen Verantwortlichkeit der einer Straftat beschuldigten Person beantwortet allerdings letztlich allein das Gericht. Dabei kann es sich durch in erster Linie Psychiater oder Psychologen sachverständig beraten lassen. Zu diesem Zweck können durch das Gericht Schuldfähigkeitsgutachten eingeholt werden.

Paraphile Störungen können dem vierten Eingangsmerkmal, der SASA, zugeordnet werden und stellen dabei die Frage einer erheblichen Verminderung der Steuerungsfähigkeit zur Diskussion, was rechtlich eine Dekulpation im Sinne des $\S 21$ StGB zur Folge haben kann (Schreiber und Rosenau 2015). Für den Begutachteten kann das Ergebnis der Schuldfähigkeitsbegutachtung bzw. der richterlichen Entscheidung erhebliche Folgen nach sich ziehen. Einerseits kann eine Minderung der Strafe die Folge sein, andererseits aber auch eine mögliche Unterbringung in einem psychiatrischen Krankenhaus nach $\S 63$ StGB, was unter bestimmten Umständen eine zeitlich zunächst nichtbegrenzte Unterbringung bedeutet. Neben paraphilen Störungen können selbstverständlich auch andere psychiatrische Störungsbilder u. a. aus dem Spektrum der Intelligenzminderungen, Schizophrenien oder auch hirnorganischen Schädigungen mit Sexualdelinquenz im Zusammenhang stehen.

Die Prävalenzraten von Paraphilien fallen je nach Stichprobe heterogen aus. Für die Allgemeinbevölkerung finden sich folgende Lebenszeitprävalenzen für mindestens einmalige paraphile Verhaltensweisen (nicht $\mathrm{zu}$ verwechseln mit Störungen): irgendeine paraphile Verhaltensweise $25 \%$ (die folgenden Verhaltensweisen sind darin enthalten), sexuell sadistische 2,7\%; pädophile $0,9 \%$ und exhibitionistische $4,3 \%$ (Baur et al. 2016). Besonders aussagekräftig ist die Untersuchung einer österreichischen Population von 1346 männlichen inhaftierten Sexualstraftätern (Eher et al. 2019). Dabei zeigten irgendeine paraphile Störung 43,3\%

\footnotetext{
${ }_{1}^{1}$ Dieser Begriff sollte obsolet sein. Gemeint sind relevante Intelligenzminderungen.

2 Auch der Begriff Abartigkeit sollte längst nicht mehr verwendet werden. Im Allgemeinen geht es um schwere Persönlichkeitsstörungen oder um schwere paraphile Störungen.
}

(die folgenden Störungen darin enthalten), eine sexuell sadistische Störung 4,4\%; eine pädophile Störung 34,5\% und einen Exhibitionismus 2,8\% der Untersuchten. Auf unsere Fragestellung bezogen bedeutet das, dass hinsichtlich der Begutachtung der Schuldfähigkeit v.a. die pädophile, die sexuell sadistische und die exhibitionistische Störung von Bedeutung sind. Im Jahre 2009 waren 12\% der Insassen psychiatrischer Krankenhäuser im Sinne des § 63 StGB wegen eines Sexualdeliktes untergebracht. Im Jahr 1995 lag dieser Anteil hingegen noch bei $21 \%$ (Traub und Weithmann 2014).

\section{Bisherige Lösungsansätze zur Einschätzung paraphiler Störungen}

Fragen im Zusammenhang mit der Begutachtung der Schuldfähigkeit bei Sexualdelikten vor dem Hintergrund einer möglicherweise damit in Verbindung stehenden paraphilen Störung sind für die damit befassten Professionen der Juristen, psychologischen und psychiatrischen Sachverständigen seit jeher eine Herausforderung.

Die vorhandenen Beurteilungskriterien der letzten ca. 30 Jahre, bezogen auf das Eingangskriterium der SASA, fokussieren auf heterogene psychische Störungsbilder, primär allerdings auf Persönlichkeitsstörungen und bisher nicht ausschließlich auf paraphile Störungen (Rasch 1999; Saß 1985; Schmidt 2008). Die sog. Mindestanforderungen für die Schuldfähigkeitsbegutachtung (Boetticher et al. 2007), die von einer interdisziplinären Expertengruppe verfasst wurden, orientieren sich an aus heterogenen Quellen differierender theoretischer Modelle stammenden Kriterien (z. B. Saß 1985) und den Auswirkungen von Persönlichkeitspathologien auf die Steuerungsfähigkeit. Diese Mindestanforderungen haben zwar gut nachvollziehbar die formalen Anforderungen an die Gutachtenerstellung dargestellt. Bei dem Versuch, die Kriterien für eine schwere paraphile Störung oder aber Merkmale für eine relevante Steuerungsminderung zu definieren, sind aber über die Kompromisse, die offensichtlich innerhalb der erstellenden Arbeitsgruppe gemacht werden mussten, theoretische Inkonsistenzen und nicht genauer operationalisierte Kriterien entstanden, die in der Folge neue Probleme aufwerfen. Diese Kriterien illustrieren psychische Zustände und deren Auswirkungen sowie äußerlich beobachtbare Verhaltensweisen.

In Bezug auf die Einschätzung des Schweregrades der paraphilen Störung (SASA) folgen die Mindestanforderungen der Hamburger Schule in der Folge von Hans Giese und Eberhard Schorsch (Kröber 2020), ohne das explizit zu benennen. Bei Giese (1962) heißen die Merkmale Leitsymptome für eine krankheitswertige Perversion (in der damaligen Terminologie). Dabei gibt es eine Mischung von 
beobachtbaren Verhaltensweisen (unten abgekürzt mit V) und psychischen Zuständen (unten abgekürzt mit P), die letztlich nach der Anamnese und mit Rückgriff auf Angaben des Probanden durch den Untersucher eingeschätzt werden müssen.

- Verfall an die Sinnlichkeit (gemeint ist die Bedeutung, die einzelne sinnliche Erfahrungen wie z.B. der Geruch bekommen, also letztlich so etwas wie eine Fetischisierung; $\mathrm{P}$ ),

- zunehmende Frequenz (V) und abnehmende Satisfaktion (P),

- Promiskuität und Anonymität (V),

- Ausbau von Fantasie (P), Praktik (V), Raffinement (V),

- süchtiges Erleben $(\mathrm{P})$,

- Periodizität der dranghaften Unruhe (V, P).

Schorsch (1971) prüfte Gieses Kriterien zunächst empirisch und fand wesentliche Bestätigungen für die v.a. phänomenologisch, normativ oder von der anthropologischen Psychiatrie herrührenden Konzeption der Leitsymptome. Später distanzierte er sich allerdings deutlich von Gieses Konzeption, indem er versuchte, mithilfe der psychoanalytischen Theorie unbewusste Motive bei Sexualstraftaten verstehbarer zu machen, die Taten als Symptome gescheiterter Abwehr zu interpretieren, bei der konflikthaftes Erleben nicht mehr nur intrapsychisch abgewehrt werden kann, sondern externalisiert wird und damit auf die Ebene des Handelns durchbricht. Schorschs (1988) Kritik und Erweiterungen der Konzepte von Giese kann man nur vor dem Hintergrund psychodynamischer Überlegungen nachvollziehen. Die progrediente Entwicklung einer paraphilen Störung als zunehmenden Zusammenbruch psychischer Abwehrmechanismen, die Schorsch den sog. sexuellen Impulshandlungen gegenüberstellte, ist insofern eigentlich nicht oder kaum verständlich, wenn die dahinter liegende psychodynamische Theorie sog. primitiver Abwehrmechanismen, d.h. Spaltung, Projektion und projektive Identifizierung, nicht gelernt worden ist und auch als Theorie geteilt wird. Es geht eben beispielsweise nicht um eine zunehmende Schwere der Taten oder um zunehmende Häufigkeit, auch wenn das häufig so interpretiert wird. Auch Kröber (2020) könnte irren, wenn er meint, dass das Hamburger Modell ,den Eindruck erweckt, als bemächtige sich die Störung eines Menschen, der an sich psychisch ungestört sein mag und nur einmal ein Fehlverhalten als angenehm und beruhigend erlebt hat, das er dann wiederholt einsetzt und das ihm schließlich über den Kopf wächst." Es geht bei der einen Gruppe eben zentral um das Ausmaß der Spaltung und die Frage, wie stark der Rest der äußerlich erkennbaren psychischen Funktion durch die paraphile Symptomatik ,entlastet“ ist, und ob das auf Dauer stabil funktioniert oder nicht. Schorsch versteht das Ausagieren gegen (eine) andere Person(en) als Scheitern dieser Ab- wehrfunktion. Mit heutigen Worten: Es gelingt nicht, paraphile Sexualität innerhalb einer Beziehung oder durch andere nichtschädigende Handlungen umzusetzen oder aber auf der Fantasieebene (z.B. begleitet von Selbstbefriedigung) zu belassen. Stattdessen muss eine destruktive Handlung gegenüber einer anderen Person durchgeführt werden.

Die Personen, die laut Schorsch in die zweite Gruppe gehören, die mit sog. Impulshandlungen, entsprechen hingegen weit mehr dem, was wir heute typischerweise als Menschen mit Borderline- oder antisozialen Persönlichkeitsstörungen erkennen und beschreiben würden. Auch Schorsch beschrieb diese Gruppe bereits als äußerlich sichtbar auffälliger.

Die beschriebenen Konzepte sind in der Folge auch im Kriterienkatalog der Mindestanforderungen für die Schuldfähigkeitsbegutachtung (Boetticher et al. 2007) von prominenter Bedeutung. Zunächst soll das Vorliegen einer Diagnose gemäß ICD- bzw. DSM-Kriterien geprüft werden. Im nächsten Schritt muss der Schweregrad der Störung eingeschätzt werden. Es sollen die Intensität der Paraphilie, die Integration in die Persönlichkeit und die bisherige Fähigkeit zur Kontrolle über paraphile Impulse eingeschätzt werden. Dabei sollen folgende Kriterien Anhalt dafür sein, dass eine paraphile Störung als schwer bezeichnet werden kann:

- Die Paraphilie bestimmt weitgehend die Sexualität des Probanden

- oder sie wird als Ich-fremd (= Ich-dyston) wahrgenommen und damit ausgeblendet.

- Sie ist in ihrer Dynamik progredient, d.h., der Proband fühlt sich von seinen paraphilen Impulsen überflutet: Sie drängen zur Umsetzung auf die Verhaltensebene.

- Dem Probanden stehen (aufgrund von bestimmten Persönlichkeitsfaktoren und/oder sexuellen Funktionsstörungen) keine bzw. kaum andere Möglichkeiten der sexuellen Befriedigung zur Verfügung.

Ohne ein entsprechendes theoretisches Modell im Hintergrund besteht allerdings ein hohes Risiko, dass diese Kriterien kataloghaft abgearbeitet werden, dies zu Missverständnissen, einer geringen Beurteilerübereinstimmung und Fehlinterpretationen führt.

Der zweite Schritt der Beurteilung, also die Einschätzung der Steuerungsfähigkeit, orientiert sich nun allerdings außer bei dem Kriterium ,konflikthafte Zuspitzung und emotionale Labilisierung vor der mutmaßlichen Tat mit bereits länger anhaltender triebdynamischer Ausweglosigkeit“ (P) nahezu ausschließlich an der Verhaltensebene (V) (Tab. 1). Das Vorgehen bleibt damit hinsichtlich der Theorien unentschieden, weil es einerseits wiederum psychodynamische Ansätze aufgreift (triebdynamische Ausweglosigkeit) und andererseits kriminologische Verhaltensmerkmale aufzählt, die auf Steuerung im Sinne der Verhaltenskontrolle hinweisen könnte. Fragen motivationaler Einschränkung 
Tab. 1 Hinweise für oder gegen erhebliche Minderung der Steuerungsfähigkeit

\begin{tabular}{ll}
\hline Hinweise für eine erhebliche Steuerungsverminderung & Hinweise gegen eine erhebliche Steuerungsverminderung \\
\hline $\begin{array}{l}\text { Konflikthafte Zuspitzung und emotionale Labilisierung vor der mut- } \\
\text { maßlichen Tat mit bereits länger anhaltender triebdynamischer Aus- } \\
\text { weglosigkeit (P) }\end{array}$ & $\begin{array}{l}\text { Hinweise auf Tatvorbereitungen und ein planmäßiges Vorgehen, wobei } \\
\text { dabei ausdrücklich nicht Ausgestaltungen in der (paraphilen) Fantasie } \\
\text { gemeint sind (V) }\end{array}$ \\
$\begin{array}{l}\text { Tatdurchführung auch in sozial stark kontrollierten Situationen (V) } \\
\text { Abrupter, impulshafter Tatablauf (V) }\end{array}$ & $\begin{array}{l}\text { Fähigkeit zu warten und/oder ein lang hingezogenes Tatgeschehen (V) } \\
\text { Komplexer Tatablauf in Etappen (V) }\end{array}$ \\
$\begin{array}{l}\text { Archaisch destruktiver Tatablauf, ritualisiert wirkend, Außenreize } \\
\text { wirken ausgeblendet (V) }\end{array}$ & Vorsorge gegen Entdeckung (V) \\
$\begin{array}{l}\text { Konstellative Faktoren (Substanzintoxikation, komorbide Störungen, } \\
\text { v. a. Persönlichkeitsstörungen, Intelligenzminderung) }\end{array}$ & Möglichkeit, in vergleichbaren Situationen anders zu handeln (V) \\
\hline
\end{tabular}

der Steuerungsfähigkeit (vergleiche Fuß et al. 2020; Kröber 2020) und Desaktualisierungsfähigkeit bleiben quasi undiskutiert.

\section{Neuere Vorschläge und empirische Untersuchungen}

Briken und Müller (2014) schlugen vor dem Hintergrund der aufgezeigten Problemlage vor einigen Jahren zunächst Kriterien vor, die inhaltlich Schweregrad und Steuerungsfähigkeit abbilden könnten, durch ihre Verwendung in Prognoseinstrumenten aber bereits standardisiert waren und daher möglicherweise die Beurteilerübereinstimmung bei der Einschätzung einer SASA und der hierdurch beeinträchtigten Steuerungsfähigkeit verbessern könnten. Es ging dabei allerdings daher gerade nicht um die Verwendung von für die Prognose bedeutsamen Faktoren für die Schuldfähigkeitsbegutachtung, sondern um die Verwendung bereits gut operationalisierter Kriterien, die sich auch für die Schuldfähigkeitsbegutachtung eignen. Briken und Müller wählten dazu 8 Items (für die SASA: paraphile sexuelle Interessen, sexuelle Dranghaftigkeit/Überbeschäftigung, Sex als Coping-Strategie, Defizite, stabile Beziehungen aufzubauen; allgemeine soziale Zurückweisung/Einsamkeit; für die Steuerungsfähigkeit: paraphile sexuelle Dranghaftigkeit, emotionaler Zusammenbruch, Zusammenbruch sozialer Unterstützung) aus zwei etablierten standardisierten Prognoseverfahren für Sexualstraftäter aus: dem STABLE2007 (Matthes und Rettenberger 2008a) und dem ACUTE2007 (Matthes und Rettenberger 2008b).

Diese wurden in einem nächsten Schritt von Brunner et al. (2016) hinsichtlich ihrer Anwendbarkeit und Interrater-Reliabilität (IRR) untersucht und weiter ausgearbeitet. Diese Pilotstudie zeigte, dass die Kriterien gut anwendbar sind. Neben einer geringen Stichprobengröße machte jedoch u. a. die fehlende Einbeziehung der Expertise von juristischer Seite weitere Forschung als nächsten Schritt notwendig.

Mit den folgenden Studien wurde angestrebt, die Qualität und Transparenz von Begutachtungen zu erhöhen und somit einen substanziellen Mehrwert für die Praxis der Schuldfähigkeitsbegutachtung zu erzielen. In der ersten Studie (Dobbrunz et al. 2020a) wurde zunächst die Beurteilerübereinstimmung (IRR) bei Verwendung der beiden Kriterienkataloge (Boetticher et al. 2007; Briken und Müller 2014) untersucht. Eine Gruppe $(N=14)$ in der Schuldfähigkeitsbegutachtung erfahrener Experten aus den 3 Berufsgruppen Psychologen, Psychiater und Juristen nahmen an der Untersuchung teil und schätzen 2 prototypische Fallvignetten ein. Die IRR der Kriterien von Briken und Müller (2014) fiel dabei höher aus als jene der Kriterien von Boetticher et al. (2007). Bei immerhin 13 der insgesamt 22 Items, bezogen auf beide Kriterienkataloge, war die IRR ungenügend; dies traf auf 12 Kriterien von Boetticher et al. (2007) und auf eines der Briken-und-Müller-Kriterien (2014) zu. Die IRR, bezogen auf das Kriterium SASA (unter Einbeziehung beider Kriterienkataloge), lag in einem moderaten bzw. substanziellen Bereich. Die IRR des Kriteriums erhebliche verminderte Steuerungsfähigkeit (ebenfalls unter Einbeziehung beider Kriterienkataloge) fiel moderat aus, jedoch waren nur 3 der 28 Beurteilungen (14 Personen beurteilten 2 Fallvignetten) voneinander abweichend, was einen Anteil von 10,7\% ausmacht. Die Auswertung der subjektiven Wichtigkeit der Kriterien beider Beurteilungsskalen zeigte, dass alle Kriterien als mindestens durchschnittlich ausschlaggebend für die Einschätzung bewertet wurden.

In der Folgestudie (Dobbrunz et al. 2020b) wurde die prädiktive Validität der beiden Kriterienkataloge hinsichtlich der SASA und der erheblich verminderten Steuerungsfähigkeit bei der Beurteilung der Fallvignetten aus der vorherigen Studie (Dobbrunz et al. 2020a) (also nicht anhand eines realen Falles) untersucht. An dieser online basierten Untersuchung nahmen 349 Probanden aus den 3 Berufsbezeichnungen Psychologie, Psychiatrie und Juristen teil. Fallvignetten und Kriterienkataloge wurden in einem randomisierten Design jeweils zufällig zugewiesen. Die Ergebnisse zeigen, dass der Kriterienkatalog von Briken und Müller (2014) sowohl die Schwere der Störung (SASA) als auch die erheblich verminderte Steuerungsfähigkeit etwa doppelt so gut vorhersagt wie der Kriterienkatalog von Boetticher et al. (2007). Die Variablen Erfahrungsgrad und Berufszu- 
gehörigkeit zeigten keine signifikanten Unterschiede - mit anderen Worten sagte also weder der Erfahrungsgrad in Sachen der Schuldfähigkeitsbegutachtung noch die Profession etwas über die Güte der Vorhersage der formulierten Fragen aus (Dobbrunz et al. 2020c). Die Analyse erbrachte auch den Befund, dass das Item ,konflikthafte Zuspitzung und emotionale Labilisierung vor der verfahrensgegenständlichen Tat" aus dem Kriterienkatalog von Boetticher et al. (2007) einen signifikanten Einfluss auf die korrekte Einschätzung der erheblich verminderten Steuerungsfähigkeit hatte. Dabei handelt es sich um ein Item, das wie oben dargestellt der psychodynamischen Theorie entstammt und den psychischen Befund des Probanden einschätzt, das dadurch wiederum in der Untersuchung besonders fehleranfällig sein könnte. Die Auswertung der subjektiven Wichtigkeit der Kriterien beider Beurteilungsskalen zeigte schließlich, dass alle Kriterien als mindestens durchschnittlich ausschlaggebend für die Einschätzung der Beweisfragen beurteilt wurden.

\section{Expertendiskussion}

Im August 2020 fand eine Expertendiskussion zum Thema Schuldfähigkeitsbegutachtung bei paraphilen Störungen nach Präsentation der vorläufigen, oben genannten Ergebnisse statt. Neben dem Richter Matthias Koller (Göttingen) nahmen der Psychologe Andreas Mokros (Hagen) und die forensische Psychiaterin Nahlah Saimeh (Düsseldorf) sowie die forensischen Psychiater Hans-Ludwig Kröber (Berlin), Jürgen L. Müller (Göttingen) und Peer Briken (Hamburg; auch Sexualmedizin) an der Onlinevideokonferenz teil. Im Folgenden sollen die dort diskutierten Themenbereiche und der dazugehörige Zwischenstand der Diskussion kurz dargestellt werden:

I. Beurteilung der bisherigen Mindestanforderungen bzw. Kriterien für die SASA und Steuerungsfähigkeit/Vor- und Nachteile/Probleme. Es wurde festgestellt, dass die Prüfung der Konzepte der SASA und der Steuerungsfähigkeit einerseits eigentlich klar voneinander zu trennen seien. Es gebe andererseits immer wieder die Tendenz, von einer vorhandenen SASA direkt auf eine erheblich verminderte Steuerungsfähigkeit zu schließen. Die Frage an die Psychowissenschaften sei allerdings, ob man tatsächlich die Anhaltspunkte für eine Schwere der Störung von den Anhaltspunkten für die Erheblichkeit ihrer Auswirkungen auf das Steuerungsvermögen unterscheiden und trennen könne, oder ob diese vom Gesetzestext nahegelegte Unterscheidung der Wirklichkeit überhaupt entspreche. Die Frage an die Juristen sei, ob Schwere und Erheblichkeit von Rechts wegen tatsächlich scharf voneinander abgegrenzt werden müssten.
Die klinische bzw. praktische Handhabbarkeit des Kriterienkatalogs sowie der Einsatz in der Breite wurden als wichtig angesehen. Neben dem oben genannten Problem der mangelnden Operationalisierung der Kriterien von Boetticher et al. (2007) sowie der Heterogenität der zugrunde liegenden Theorien wurden die unterschiedlichen Beurteilungsmaßstäbe in verschiedenen Bundesländern, lokalen Kulturen und Erfahrungshintergründen der Richter markiert.

II. Beurteilung des bisherigen empirischen Vorgehens. Die empirische Untersuchung der Reliabilität und Validität der beiden Kriterienkataloge wurde auch durch die Teilnehmenden, die nicht an der Konzeption mitgewirkt haben (alle außer JLM und PB) als wichtig und notwendig erachtet. Allerdings erfolgte der Hinweis zu überprüfen, ob es neben den Items aus dem Prognoseverfahren Stable-2007 (Matthes und Rettenberger 2008a) noch andere Konstrukte bzw. Items gibt, die bisher nicht ausreichend berücksichtigt worden sind.

III. Verhältnis von paraphiler Störung und Persönlichkeitsstörung. Kontrovers blieb die Frage zum Verhältnis von paraphiler Störung und Persönlichkeitsstörung. Persönlichkeit (resp. Persönlichkeitsstörung) und Sexualität (hier: paraphile Störung) sind zweifellos eng miteinander verwoben, sodass eine Trennung einerseits künstlich erscheint. Allerdings gebe es auch Konstellationen, in denen die Persönlichkeit nicht krankheitswertig gestört wirke, die paraphile Störung dennoch stark ausgeprägt anmute. Sowohl das Konzept des Strukturniveaus bzw. der strukturellen Störung als auch das Konzept der Desaktualisierung (im Sinne der Unterdrückung von Bedürfnissen, Impulsen, motorischen Reaktionen, aufschieben und verzichten können; Janzarik 2004; Janzarik in diesem Heft sowie Fuß et al. 2020) haben sowohl in den Kriterien von Boetticher et al. (2007) als auch in denen von Briken und Müller (2014) bisher offensichtlich nicht ausreichend Berücksichtigung gefunden. Nicht ausreichend gewürdigt werde in bisherigen Ansätzen die motivationale Steuerungsfähigkeit (Kröber 2020), also ,die Fähigkeit, das eigene Handeln auch bei starken Wünschen und Bedürfnissen normgerecht zu kontrollieren und die Ausführung normwidriger Motivationen zu inhibieren“.

IV: Verhältnis von paraphiler Störung und hypersexueller bzw. zwanghafter sexueller Verhaltensstörung/ist eine relevante Steuerungsminderung auch bei zwanghafter sexueller Verhaltensstörung ohne paraphile Störung möglich? Prinzipiell war eine solche Konstellation für Teilnehmende der Diskussion denkbar. Ein zentraler Aspekt der Frage scheint neben der Quantität der sexuellen Symptomatik dennoch die Qualität der sich daraus ergebenden psychi- 
schen Einschränkungen zu sein. Zudem sei es sinnvoll zu überprüfen, ob hypersexuelle Phänomene auch sozialverträglich auslebbar sind.

\section{Schlussbemerkungen und Ausblick}

Zusammenfassend erscheint es bei der Begutachtung der Schuldfähigkeit von Personen mit paraphilen Störungen gegenwärtig sinnvoll, das Vorgehen der operationalisierten, kriteriengeleiteten Unterstützung der Begutachtung (im Sinne von Briken und Müller 2014) weiter in der Forschung zu verfolgen und für eine Transparenz ggf. auch schon aktuell in den Beurteilungsprozess einzubeziehen. Die vorgeschlagenen Kriterien sind im Begutachtungsprozess jedoch keineswegs als alleinige Beurteilungsquelle anzusehen. Das Vorgehen sollte auch nicht so verstanden werden, dass Prognosekriterien für die Einschätzung des Schwergrades der Störung und für die Steuerungsfähigkeit genutzt werden können oder sollen. Schließlich ersetzt das Vorgehen weder die ausführliche Anamnese und Exploration noch ein intensives Aktenstudium. Eine weitere Auseinandersetzung mit dieser Thematik in der Forschung wird angestrebt, u. a. in Form von interdisziplinären Arbeitstreffen und weiteren empirischen Untersuchungen. Dabei wird von Bedeutung sein, die Begriffe der SASA und Steuerungsfähigkeit juristisch zu definieren und die Definition in die psychiatrisch-psychologischen Disziplinen zu übersetzen. Sinnvoll erscheint die Auseinandersetzung mit den theoretischen Grundlagen der Einschätzung dessen, was psychiatrisch-psychologisch eingeschränkte Steuerungsfähigkeit bedeutet und ausmacht (z. B. die Frage, wie motivationale Steuerungsfähigkeit theoretisch hergeleitet wird). Aus dem bisherigen Vorgehen ist deutlich geworden, dass weitere Kriterien in der Beurteilung Berücksichtigung finden müssen - so z.B. das Strukturniveau oder die Desaktualisierungsfähigkeit. Gleichzeitig stellt sich hier die Frage nach dem geeigneten theoretischen Modell. Schließlich ist die Frage, ob nur das Merkmal der paraphil gestörten Sexualität geeignet ist, um krankheitswertige von nichtkrankheitswertiger Sexualität abzugrenzen, oder ob nicht vergleichbar auch die zwanghafte sexuelle Verhaltensstörung im Sinne der neuen ICD-11 prinzipiell für eine relevante Beeinträchtigung infrage kommt.

Danksagung Wir danken Matthias Koller (Göttingen), Andreas Mokros (Hagen), Nahlah Saimeh (Düsseldorf), Hans-Ludwig Kröber (Berlin) und Jürgen L. Müller (Göttingen) für die Teilnahme an der Expertenkommission und die dort getätigten, hilfreichen Hinweise.

Funding Open Access funding enabled and organized by Projekt DEAL.

Interessenkonflikt S. Dobbrunz und P. Briken geben an, dass kein Interessenkonflikt besteht.
Open Access Dieser Artikel wird unter der Creative Commons Namensnennung 4.0 International Lizenz veröffentlicht, welche die Nutzung, Vervielfältigung, Bearbeitung, Verbreitung und Wiedergabe in jeglichem Medium und Format erlaubt, sofern Sie den/die ursprünglichen Autor(en) und die Quelle ordnungsgemäß nennen, einen Link zur Creative Commons Lizenz beifügen und angeben, ob Änderungen vorgenommen wurden.

Die in diesem Artikel enthaltenen Bilder und sonstiges Drittmaterial unterliegen ebenfalls der genannten Creative Commons Lizenz, sofern sich aus der Abbildungslegende nichts anderes ergibt. Sofern das betreffende Material nicht unter der genannten Creative Commons Lizenz steht und die betreffende Handlung nicht nach gesetzlichen Vorschriften erlaubt ist, ist für die oben aufgeführten Weiterverwendungen des Materials die Einwilligung des jeweiligen Rechteinhabers einzuholen.

Weitere Details zur Lizenz entnehmen Sie bitte der Lizenzinformation auf http://creativecommons.org/licenses/by/4.0/deed.de.

\section{Literatur}

Baur E, Forsman M, Santtila P (2016) Paraphilic sexual interests and sexually coercive behavior: a population-based twin study. Arch Sex Behav 45:1163-1172

Boetticher A, Nedopil N, Bosinski HAG et al (2007) Mindestanforderungen für Schuldfähigkeitsgutachten. Forens Psychiatr Psychol Kriminol 1:3-9

Briken P, Müller JL (2014) Beurteilung der Schuldfähigkeit bei paraphiler Störung. Nervenarzt 85:304-311

Brunner F, Müller JL, Vogel S et al (2016) Evaluation von operationalisierten Kriterien zur Schuldfähigkeitsbeurteilung bei paraphiler Störung. Recht Psychiatr 34:228-236

Dobbrunz S, Daubmann A, Müller JL et al (2020a) Interrater-Reliabilität der kriteriengeleiteten Beurteilung der Schuldfähigkeit bei paraphilen Störungen. Nervenarzt. https://doi.org/10.1007/s00115020-00920-1

Dobbrunz S, Daubmann A, Müller JL et al (2020b) Predictive validity of operationalized criteria for the assessment of criminal responsibility of sex offenders with paraphilic disorders. (eingereicht)

Dobbrunz S, Daubmann A, Müller JL et al (2020c) Der Einfluss von Profession und Erfahrung hinsichtlich der kriteriengeleiteten Beurteilung der Schuldfähigkeit bei paraphilen Störungen. (in Vorbereitung)

Eher R, Rettenberger M, Turner D (2019) The prevalence of mental disorders inincarcerated contact sexual offenders. Acta Psychiatr Scand 139:572-581

Fuß J, Voulgaris A, Briken P (2020) Schuldfähigkeit bei Personen, die wegen Nutzung von Missbrauchsabbildungen angeklagt sind. Forens Psychiatr Psychol Kriminol. https://doi.org/10.1007/s11757020-00624-x

Giese H (1962) Psychopathologie der Sexualitat. Enke, Stuttgart

Janzarik W (2004) Autopraxis, Desaktualisierung, Aktivierung und die Willensthematik. Nervenarzt 75:1035-1060

Jescheck H-H, Weigend T (1996) Lehrbuch des Strafrechts. Allgemeiner Teil, 5. Aufl. Duncker \& Humblot, Berlin

Kröber H-L (2020) Konzepte und Implikationen der verminderten Schuldfähigkeit. Forens Psychiatr Psychol Kriminol. https://doi. org/10.1007/s11757-020-00626-9

Matthes A, Rettenberger M (2008a) Die deutsche Version des STABLE-2007 zur dynamischen Kriminalprognose bei Sexualstraftätern. Institut für Gewaltforschung und Prävention, Wien

Matthes A, Rettenberger M (2008b) Die deutsche Version des ACUTE2007 zur dynamischen Kriminalprognose bei Sexualstraftätern. Institut für Gewaltforschung und Prävention, Wien

Rasch W (1999) Forensische Psychiatrie, 2. Aufl. Kohlhammer, Stuttgart 
Saß H (1985) Ein psychopathologisches Referenzsystem für die Beurteilung der Schuldfähigkeit. Forensia 6:33-43

Schmidt AF (2008) Psychologische Schuldfähigkeitsbegutachtung bei sogenannter schwerer anderer seelischer Abartigkeit - eine Begutachtungsheuristik auf empirischer Grundlange. Centaurus, Herbolzheim

Schorsch E (1971) Sexualstraftäter. Enke, Stuttgart

Schorsch E (1988) Affekttaten und sexuelle Perversionstaten im strukturellen und psychodynamischen Vergleich. In: Der Mensch in der Psychiatrie. Springer, Berlin, Heidelberg, S 344-358
Schreiber HL, Rosenau H (2015) Rechtliche Grundlagen der psychiatrischen Begutachtung. In: Venzlaff U, Förster K, Dreßing H, Habermeyer E (Hrsg) Psychiatrische Begutachtung: Ein praktisches Handbuch für Ärzte und Juristen, 6. Aufl. Urban \& Fischer, München, S 89-143

Traub HJ, Weithmann G (2014) Gemeinsame Entwicklung, unterschiedliche Inzidenz. Forens Psychiatr Psychol Kriminol 8:199-207 